\title{
On-the-fly temperature-dependent cross section treatment under extremes in RMC code
}

\author{
Lei Zheng ${ }^{1, *}$, Wei Wang ${ }^{1}$, and Kan Wang ${ }^{1}$ \\ ${ }^{1}$ Department of Engineering Physics, Tsinghua University, 1st Qinghuayuan, Haidian District, Beijing, 100084, China
}

\begin{abstract}
Neutron transport relevant to inertial confinement fusion always involves extremes, in which the physical quantities are extremely high, widely distributed and changes rapidly with space and time. In order to solve the memory and efficiency problems in nuclear data storage and processing, the on-the-fly temperaturedependent cross section treatment technique was investigated and developed under extremes in RMC code. Different strategies were adopted for different energy regions, i.e., the free gas model in the thermal region, the TMS method with $0 \mathrm{~K}$ basis cross section temperature in the resolved resonance region, and the infinite dilution cross section in the unresolved resonance region, whereas the high energy region above the unresolved resonance region was not treated currently. The test results of Godiva sphere and plutonium sphere show that the on-the-fly technique has high accuracy, but the efficiency of the proposed technique still needs to be improved for some cases, and the optimization work with the elevated basis cross section temperatures is ongoing.
\end{abstract}

\section{Introduction}

Neutron transport relevant to inertial confinement fusion always involves extremes, in which the physical quantities are extremely high, widely distributed and changes rapidly with space and time. The temperature of the background material can be as high as 100-million Kelvin, and the maximum velocity of the boundary motion can reach 1million meters per second. Moreover, both of the background temperature and the boundary motion velocity change rapidly with space and time in a wide range. Since the neutron cross section is a function of the neutron speed relative to the target nuclei, affected by the thermal motion of the target nuclei and the boundary motion, the traditional pre-generated cross section method [1] is invalid under extremes because of the memory limitation of computers. Trumbull [2] suggested that ACE dataset at every $10 \mathrm{~K}$ could provide the accuracy need for simple interpolation between temperature points, considering the temperature range from room temperature to 100-million Kelvin, the amount of ACE data could reach about 11000TB, which is unacceptable for even super computers. Meanwhile, the generation of such large amount of dataset is very time consuming. In order to solve the memory and the efficiency problems in data storage and processing, the onthe-fly treatment of cross section is the right choice under extremes.

Science the consideration of the effect of both thermal motion and boundary motion on Doppler broadening is too complicated, this paper mainly focus on the temperature effect, the boundary motion effect will be taken into consideration in the future work. The on-the-fly temperaturedependent cross section treatment strategy in the continu-

\footnotetext{
*e-mail: zl17@mails.tsinghua.edu.cn
}

ous energy Reactor Monte Carlo code RMC [3] was investigated and developed under extremes. Detailed description of the methods and the validation results are presented in the remainder part of this paper.

\section{Methods}

Neutron cross section as a function of the incident energy is generally divided into three regions in the conventional reactor physics, namely, the thermal region, the resonance region, and the high-energy region, while the resonance region could also be divided into resolved region and unresolved region, depending on the nuclide. Doppler broadening effect becomes pronounced with the increase of temperature, especially at high temperatures. The temperature effect on different energy regions show huge difference as shown in Fig. 1, which takes the total cross section of ${ }^{235} \mathrm{U}$ from ENDF/B-VIII.0 [4] library as an example. Therefore, different strategies are adopted for different energy regions.

\subsection{Thermal energy region}

In the thermal energy region, since the number of neutrons is extremely small, the contribution of the thermal neutrons to the transport process is not as significant as that in the conventional reactor physics. In order to simulate the temperature effect accurately, the free gas model in RMC code is adopted to account for the temperature effect in the thermal region.

\subsection{Resolved resonance region}

In the resolved resonance region (RRR), where the temperature effect is very significant, two on-the-fly strate- 


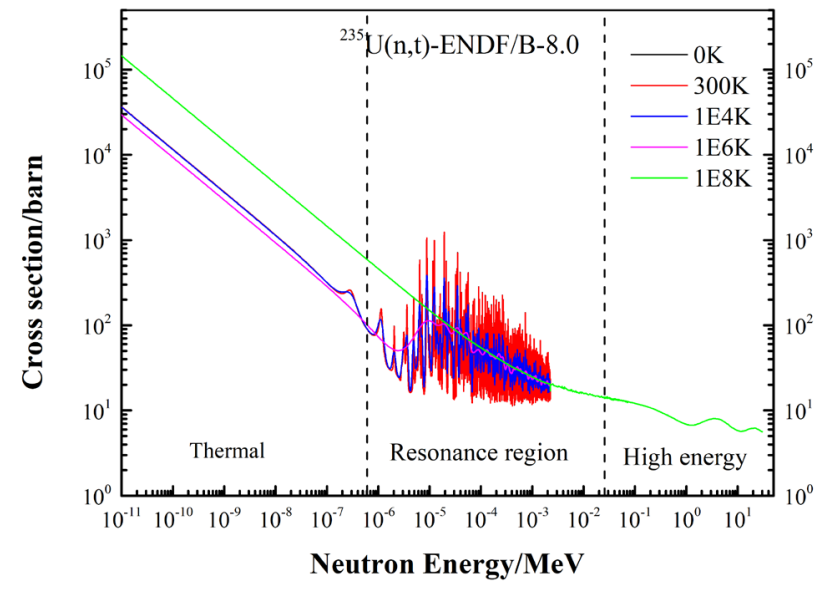

Figure 1. Temperature effect on ${ }^{235} \mathrm{U}$ total cross section from ENDF/B-VIII.0 library.

gies were developed in RMC code, the target motion sampling (TMS) [5] method and the improved GaussHermite method [6]. Since the using of the Gauss-Hermite quadrature has limitation [7], the improved Gauss-Hermite method is invalid at high temperatures for some nuclides. While the TMS method takes the thermal motion of target nuclide into account explicitly. The coordinate transform to the target-at-rest frame at each collision site and the rejection sampling technique are used to model arbitrary temperatures with only $0 \mathrm{~K}$ continuous-energy cross sections [5]. This method is a neutron tracking strategy with wide applicability at high temperatures, meanwhile, considering the combination with the boundary motion effect in the near future, TMS method is selected to handle the temperature effect in RRR, the details of this method can be referred to literatures $[5,8]$.

\subsection{Unresolved resonance region and high energy region}

In the unresolved resonance region, since the resonance peak decreases significantly, the resonance self-screen effect will disappear at high temperatures [9], the infinite dilution cross section is adopted in this paper to simulate the temperature effect approximately. For the high energy region above the URR, the temperature effect was not treated currently.

\section{Numerical results and analysis}

\subsection{Test cases}

Two cases of Godiva sphere and Plutonium sphere were modified and adopted to validate the on-the-fly technique under extremes. The Godiva sphere is a 10-layer-shell fast spectrum case, the central layer of the assembly is a high-enriched uranium (HEU) shell with an enrichment of about $93 \%$ for ${ }^{235} \mathrm{U}$, the middle part is a 4-layer HEUpolyethylene alternate shell, and the outer part is coated with HEU shell. The plutonium sphere is a 3-layer-shell soft spectrum case, the central layer is a mixture of Plutonium, polyethylene, and iron, the middle layer is a stainless steel shell, and the outer layer is a thin cadmium coating. The sketches of the two cases plotted by RMC are given in Fig. 2 and Fig. 3.

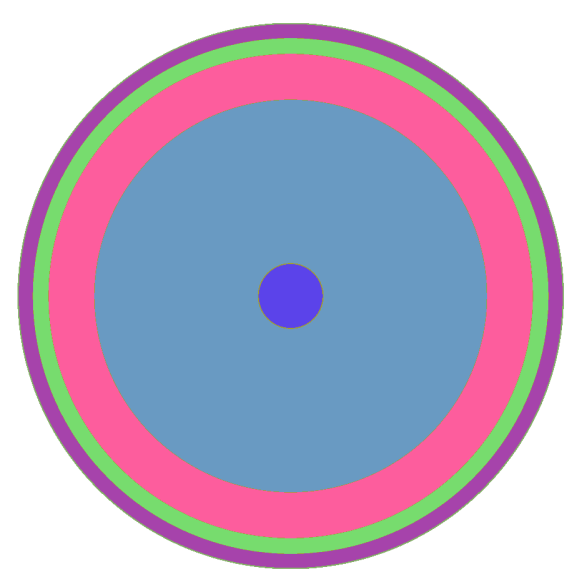

Figure 2. Godiva sphere.

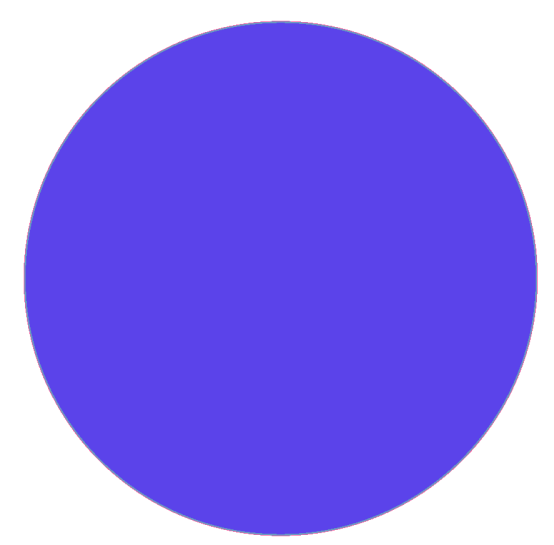

Figure 3. Plutonium sphere

In order to validate the accuracy of the on-the-fly strategy, two conditions were calculated, one is the $0 \mathrm{~K}$ cross section with TMS method, the other is the accurate cross section temperature with Doppler-broadening rejection correction (DBRC) [5], which considers the temperature effect on both cross sections and energy direction distributions. The second condition is regarded as the reference. $\mathrm{OK}$ ACE format neutron library data was taken from the ENDF/B-VII.0 [10] official site, and the accurate ACE format data at $300 \mathrm{~K}, 1000 \mathrm{~K}, 2000 \mathrm{~K}, 5000 \mathrm{~K}, 10^{4} \mathrm{~K}$, $10^{5} \mathrm{~K}, 10^{6} \mathrm{~K}, 10^{7} \mathrm{~K}$ were generated using the latest version of NJOY2016 [11] based on the ENDF/B-VII.0 neutron data library.

All the cases were calculated in the criticality mode using RMC code, version 2.5, for the Godiva sphere case, 30000 neutrons per cycle were used for total 150 cycles with 10 inactive cycles, and for the plutonium sphere case, 10000 neutrons per cycle were used for total 130 cycles 
with 30 inactive cycles. All the calculations were conducted in the serial mode, using the intel (R) core(TM) i7-7700 CPU @3.60GHz processor.

\subsection{Results and analysis}

The fission rate as a function of the neutron energy for the two cases based on the accurate library with DBRC is given in Fig.4 and Fig.5. It can be observed that the neutrons with energy higher than $100 \mathrm{keV}$ contribute to the fission rate dominatingly in the Godiva sphere. While in the plotunium sphere, neutrons in the energy range from $0.1 \mathrm{eV}$ to serval $\mathrm{keV}$ contribute to the fission rate mostly, morever, with the temperature increase, the energy of the mostly contributed neutrons increase simultaneously. These results also reveal that the tempreture effect is pronounced for the soft energy spectrum and special attention needs to be paided under extremes.

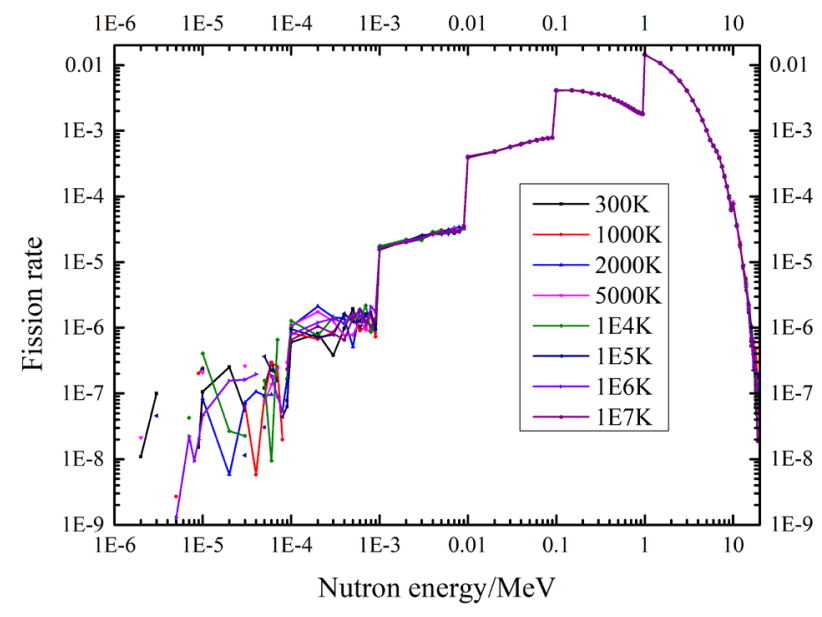

Figure 4. Fission rate distribution in Godiva sphere, layer 5.

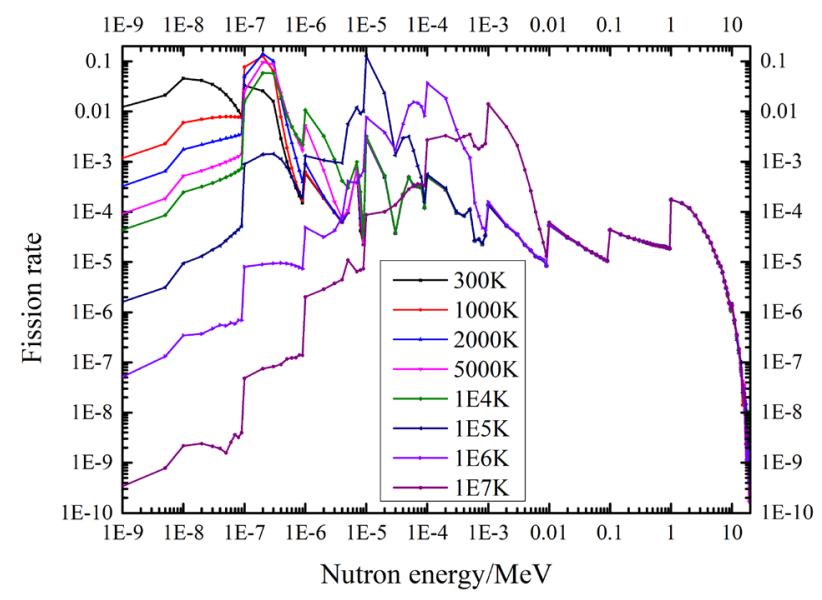

Figure 5. Fission rate distribution in Poltunium sphere.

The k-effective values, the standard deviation and the calculation time for the two cases are given in Table1 and Table2. In the comparison column, the $\sigma$ is defined as $\Delta k_{\text {eff }}$ devided by the $k_{\text {eff }}$ of the reference (the accurate library with DBRC), and $\Delta k_{e f f}$ is defined as the $k_{e f f}$ of the on-the-fly techquine subtracted by that of the reference.

It can be found that the $k_{e f f}$ of the on-the-fly techquine agrees with that of the reference within $3 \sigma$ ( confidence level $99.73 \%$ ) for both cases. For the fast spectrum Godiva case, the time consumption of the on-the-fly technique increase less than $50 \%$ compared to that of the standard method, while for the soft spectrum plutonium case, the time consumption increase with the temperature increase, at the temperature of $10^{7} \mathrm{~K}$, the time consumption increase about 30 times as that of the reference, which is unacceptable when appling the on-the-fly technique. Since this is the preliminary result, the optimization work with the elevated basis cross section temperatures is still ongoing, and will be reported in the near future.

\section{Conclusion}

The on-the-fly temperature-dependent cross section treatment technique under extremes in RMC code has been investigated and developed. The present work mainly focus on the temperature effect on neutron cross sections, and the boundary motion effect will be taken into consideration in the future work. Different strategies were adopted for different energy regions, i.e., the free gas model in the thermal region, the TMS method with 0K basis cross section temperature in RRR, and the infinite dilution cross section in URR, whereas the high-energy region above the URR was not treated currently.

The test results of Godiva sphere and plutonium sphere show that the on-the-fly technique has high accuracy compared to the reference, while the efficiency of the proposed technique still needs to be improved for some cases, and the optimization work with the elevated basis cross section temperatures is ongoing.

This work is partially supported by the Science Challenge Project (No. TZ2018001) and the National Natural Science Foundation of China (No. 11775127).

\section{References}

[1] J. Yu, J. Liang, K. Wang, Transactions of American Nuclear Society 112,663(2015)

[2] T. H. Trumbull,Nucl. Technol 156,75(2006)

[3] K. Wang, Z. Li, D. She. J. Liang, Q. Xu, Y. Qiu, J. Yu, J. Sun, X. Fan, G. Yu, Ann. Nucl. Energy 82,121(2015)

[4] D. A. Brown, M. B. Chadwick, R. Capote, A. C. Kahler, A. Trkov, M. W. Herman, A. A. Sonzogni, Y. Danon, et al. Nucl. Data Sheets 148,1(2018)

[5] S. Liu, Y. Yuan, J. Yu, K. Wang,Ann. Nucl. Energy 94,144(2016)

[6] Y. Yuan, S. Liu, Q. Xu, K. Wang, Transactions of American Nuclear Society 115,1097(2016)

[7] P. K. Romano, H. Trumbull, Ann. Nucl. Energy 75,358(2015) 
Table 1. Comparison of keff and time for the Godiva sphere

\begin{tabular}{|c|c|c|c|c|c|c|c|c|c|}
\hline \multirow{2}{*}{ Temperature/K } & \multicolumn{3}{|c|}{ Reference } & \multicolumn{3}{c|}{ On-the-fly } & \multicolumn{3}{c|}{ Comparison } \\
\cline { 2 - 10 } & $k_{\text {eff }}$ & Std. & Time/min & $k_{\text {eff }}$ & Std. & Time/min & $\Delta k_{\text {eff }}$ & $\sigma$ & Time ratio \\
\hline 300 & 1.000719 & 0.000289 & 0.6745 & 1.000723 & 0.000371 & 0.8563 & $-4 \mathrm{E}-06$ & -0.01384 & 1.27 \\
\hline 1000 & 1.001121 & 0.00027 & 0.6713 & 1.000889 & 0.000376 & 0.8548 & 0.000232 & 0.859259 & 1.27 \\
\hline 2000 & 1.00066 & 0.000305 & 0.6709 & 1.0004 & 0.000378 & 0.8721 & 0.00026 & 0.852459 & 1.30 \\
\hline 5000 & 1.001587 & 0.000322 & 0.6680 & 1.001562 & 0.000383 & 0.8622 & $2.5 \mathrm{E}-05$ & 0.07764 & 1.29 \\
\hline $10^{4}$ & 1.00069 & 0.000297 & 0.6682 & 1.000516 & 0.000337 & 0.8756 & 0.000174 & 0.585859 & 1.31 \\
\hline $10^{5}$ & 1.000079 & 0.000303 & 0.6632 & 1.000873 & 0.000366 & 0.8807 & -0.00079 & -2.62046 & 1.33 \\
\hline $10^{6}$ & 1.001402 & 0.000291 & 0.6681 & 1.000826 & 0.000381 & 0.9394 & 0.000576 & 1.979381 & 1.41 \\
\hline $10^{7}$ & 1.000797 & 0.000289 & 0.6927 & 1.000565 & 0.000359 & 1.0136 & 0.000232 & 0.802768 & 1.46 \\
\hline
\end{tabular}

Table 2. Comparison of keff and time for the plutonium sphere

\begin{tabular}{|c|c|c|c|c|c|c|c|c|c|}
\hline \multirow{2}{*}{ Temperature/K } & \multicolumn{3}{|c|}{ Reference } & \multicolumn{3}{c|}{ On-the-fly } & \multicolumn{3}{c|}{ Comparison } \\
\cline { 2 - 10 } & $k_{\text {eff }}$ & Std. & Time/min & $k_{\text {eff }}$ & Std. & Time/min & $\Delta k_{\text {eff }}$ & $\sigma$ & Time ratio \\
\hline 300 & 0.989635 & 0.000713 & 1.4956 & 0.988625 & 0.001046 & 2.8652 & -0.00101 & -1.41655 & 1.91 \\
\hline 1000 & 1.032701 & 0.000806 & 1.3337 & 1.031751 & 0.00088 & 2.5695 & -0.00095 & -1.17866 & 1.93 \\
\hline 2000 & 1.02259 & 0.000756 & 1.3834 & 1.02028 & 0.000946 & 2.6427 & -0.00231 & -3.05556 & 1.91 \\
\hline 5000 & 0.796182 & 0.000753 & 1.6717 & 0.794057 & 0.000791 & 3.7361 & -0.00212 & -2.82205 & 2.23 \\
\hline $10^{4}$ & 0.583257 & 0.000579 & 2.7292 & 0.581579 & 0.000815 & 5.7882 & -0.00168 & -2.8981 & 2.12 \\
\hline $10^{5}$ & 0.607844 & 0.000567 & 4.7721 & 0.608242 & 0.000844 & 14.5240 & 0.000398 & 0.70194 & 3.04 \\
\hline $10^{6}$ & 0.431459 & 0.000536 & 7.8892 & 0.431472 & 0.000798 & 49.8727 & $1.3 \mathrm{E}-05$ & 0.024254 & 6.32 \\
\hline $10^{7}$ & 0.143356 & 0.000161 & 13.8834 & 0.143082 & 0.000319 & 416.676 & -0.00027 & -1.70186 & 30.01 \\
\hline
\end{tabular}

[8] T. Viitanen, J. Leppanen, Nucl. Sci. Eng. 171,165(2012)

[9] D. Tian, Z. Na, C. Liu, Chinese J. Comput. Phys. 13,300(1996)

[10] M. B. Chadwick, P. Oblozinsky, M. Herman, N.M. Greene, R.D. McKnight, D.L. Smith, et al.Nucl. Data
Sheets 107,2931(2006)

[11] R. E. MacFarlane, D. W. Muir, R. M Boicourt, A. C. Kahler, J. L. Conlin, W. Haeck, LA-UR-17-20093 (2018) 